\title{
Six Critically Ill COVID-19 Pregnant Patients at a Detroit Hospital: A Case Series
}

\author{
Nicolina Smith ${ }^{1}$, Leah Hong ${ }^{1}$, Madhurima Keerthy ${ }^{2}$, Monica Lee-Griffith ${ }^{1}$, Robyn Garcia ${ }^{1}$, \\ Majid Shaman ${ }^{1}$, and Gregory Goyert ${ }^{1}$ \\ ${ }^{1}$ Henry Ford Health System \\ ${ }^{2}$ Henry Ford Hospital
}

June 1, 2020

\begin{abstract}
Objective: To highlight treatment modalities, management, and outcomes of 6 critically ill Coronavirus 2019 (COVID-19) pregnant patients. Design: We present a case series of 6 pregnant patients with severe COVID-19 infection requiring admission into the intensive care unit. Setting: Urban educational facility in Detroit, Michigan. Population: Pregnant women between the ages of 18-37 years old who tested positive for COVID-19 and required intensive care interventions. Methods: This is a retrospective, single-center case series of pregnant women who were infected with COVID-19 and subsequently required critical care. Main Outcome Measures: Maternal and fetal outcomes were measured. Results: All women required intensive care unit intervention, with 5 requiring mechanical ventilation. The average length of intubation was 5.6 days and average length of stay was 10.8 days. There was 1 full-term delivery via cesarean section and 2 preterm deliveries. All neonates tested negative for COVID-19. All patients discharged home on room air. Conclusion: This case series adds to the expanding literature describing the complex care surrounding pregnant patients with severe COVID-19 pneumonia requiring intensive care management. As the pandemic continues we hope our experience and treatment modalities can contribute to future care of patients.
\end{abstract}

Six Critically Ill COVID-19 Pregnant Patients at a Detroit Hospital: A Case Series

Nicolina Smith, DO, MBA, ${ }^{1}$ Leah Hong, MD,${ }^{1}$ Monica Lee-Griffith, MD/MBA, ${ }^{1}$ Madhurima Keerthy, MD,${ }^{1}$ Majid Shaman, MD, ${ }^{2}$ Robyn Garcia, $\mathrm{MD},{ }^{2}$ Gregory Goyert, $\mathrm{MD}^{2}$

${ }^{1}$ Department of Obstetrics and Gynecology, Henry Ford Hospital, Detroit, MI

${ }^{2}$ Department of Obstetrics and Gynecology, Division of Maternal Fetal Medicine, Henry Ford Hospital, Detroit, MI

Mailing address to all authors 2799 W. Grand Blvd. Detroit, MI 48202

Corresponding Author: Nicolina Smith,NSmith22@hfhs.org, 313-848-0620

2799 W. Grand Blvd. Detroit, MI 48202

Short Title: Critically Ill COVID-19 Pregnant Patients

Acknowledgments: We would like to acknowledge Stephanie Stebens for her assistance with preparing the manuscript for submission.

\section{Abstract}

Objective: To highlight treatment modalities, management, and outcomes of 6 critically ill Coronavirus 2019 (COVID-19) pregnant patients.Design: We present a case series of 6 pregnant patients with severe 
COVID-19 infection requiring admission into the intensive care unit.Setting: Urban educational facility in Detroit, Michigan.Population: Pregnant women between the ages of 18-37 years old who tested positive for COVID-19 and required intensive care interventions.Methods: This is a retrospective, single-center case series of pregnant women who were infected with COVID-19 and subsequently required critical care.Main Outcome Measures: Maternal and fetal outcomes were measured.Results: All women required intensive care unit intervention, with 5 requiring mechanical ventilation. The average length of intubation was 5.6 days and average length of stay was 10.8 days. There was 1 full-term delivery via cesarean section and 2 preterm deliveries. All neonates tested negative for COVID-19. All patients discharged home on room air.Conclusion: This case series adds to the expanding literature describing the complex care surrounding pregnant patients with severe COVID-19 pneumonia requiring intensive care management. As the pandemic continues we hope our experience and treatment modalities can contribute to future care of patients.

\section{Introduction}

Coronavirus-2019 (COVID-19) can cause viral pneumonia with rapid deterioration into acute respiratory distress syndrome requiring intubation. ${ }^{1}$ There have been limited case reports on critically ill pregnant patients with COVID-19. Pregnant patients with respiratory collapse secondary to COVID-19 present multiple management challenges. We present a case series of 6 pregnant patients with severe COVID-19 infection requiring admission into the intensive care unit. Their clinical course, medical management, and critical care interventions are described.

\section{Methods}

This is a retrospective, single-center case series of pregnant women who were infected with COVID-19 that subsequently required critical care. The Henry Ford Health System Institutional Review Board approved this study. After approval, data was extracted from the EPIC (EPIC systems corporation, Verona WI) electronic medical record system by medical record number after the patient was admitted to the intensive care unit (ICU) at a tertiary care center in Detroit, Michigan. Inclusion criteria were pregnant patients with a positive COVID-19 nasal swab and admission to the intensive care unit and treated between March 1, 2020 and May 1, 2020. There were no exclusion criteria. Data collected included demographics such as race, gestational age, body mass index, and comorbidities. Admission symptoms, COVID-19 exposure, chest radiography, relevant COVID-19 labs, treatment modality, and patient outcomes were all recorded.

\section{Results}

Six pregnant women with severe COVID-19 pneumonia at Henry Ford Hospital required intensive care unit hospitalization (mean age, 30 years old; range, 18-37 years old). One woman had a history of obstructive sleep apnea, well-controlled asthma, and uncontrolled type 2 diabetes mellitus; 1 had a history of hypothyroidism and hypertriglyceridemia. Four of 6 patients were morbidly obese (mean body mass index [BMI], 37.26 $\mathrm{kg} / \mathrm{m}^{2}$; range 22.37-56.0 kg/m²). Patients' race included 4 African Americans, one Hispanic, and one Arab American individual. Gestational age at time of admission ranged from 23 weeks to 37 weeks and 2 days (Table 1).

Key diagnostic findings included chest x-ray results indicative of viral pneumonia in all 6 patients; decreased absolute lymphocyte count in 4 of 6 patients tested; abnormal liver function tests in 2, and hypertriglyceridemia in 1. Clinical findings seen in all 6 of the patients included shortness of breath and tachycardia; 5 of 6 patients presented with tachypnea; 4 of 6 presented with hypoxia requiring immediate oxygen supplementation; and 2 of 6 patients had a known COVID-19 positive exposure (Table 2).

All women were treated with steroids, initially intravenous methylprednisolone $40 \mathrm{mg}$ twice a day for 3 days. A shortage quickly developed and oral prednisone 40-80 mg twice a day for 7-10 days was substituted for 4 patients. Standard treatment also included hydroxychloroquine $400 \mathrm{mg}$ oral loading dose for 2 doses and then $200 \mathrm{mg}$ oral twice daily for a total of 5 days. One patient did not receive hydroxychloroquine therapy due to prolonged corrected QT interval of greater than $500 \mathrm{~ms}$; one patient declined hydroxychloroquine due to concern for possible fetal effects; and 4 patients received a full course of hydroxychloroquine as described. 
Three of 6 patients were treated with antibiotics for superimposed bacterial pneumonia. All 6 patients received venous thromboembolism prophylaxis; 5 patients with preterm gestation received betamethasone secondary to the potential for preterm delivery (Table 3).

All 6 patients received antepartum testing with fetal non-stress tests. The 2 patients who were admitted at 23 weeks gestational age were monitored for 20 minutes once a day. The remaining 4 patients had fetal non-stress tests every 8 hours. As long as the tracing was reassuring, they would be taken off the monitor. Since our patients were sedated, we did not have expectations to have accelerations while monitoring.

There was 1 full-term delivery via cesarean section at 37 weeks and 4 days after intubation due to nonreassuring fetal heart tones remote from delivery with absent variability noted on fetal heart tracing. There were 2 preterm deliveries. One delivery was an urgent cesarean section at 36 weeks and 5 days for nonreassuring fetal status in the setting of preeclampsia with severe features and worsening respiratory compromise from COVID-19 pneumonia. The other preterm delivery was a vacuum-assisted vaginal delivery at 36 weeks and 3 days gestation following labor induction for a persistent category 2 fetal heart tracing. All neonates tested negative for COVID-19. One infant remained hospitalized for 7 days for respiratory distress and suspicion of sepsis. The other 2 infants were discharged on hospital day 2 (Table 4).

\section{Discussion}

COVID-19 has been associated with acute respiratory failure leading to acute respiratory distress syndrome. While the treatment of acute respiratory distress syndrome in pregnancy generally mirrors the non-pregnant population, it remains unclear if COVID-19 pneumonia in pregnancy has a single characteristic clinical course or is more variable. Recent literature has proposed different clinical 'phenotypes' of COVID-19 pneumonia depending on infection severity, ventilatory responsiveness, and time elapsed from onset of disease. ${ }^{1}$ Optimal intensive care interventions and ventilatory support settings require an appreciation of the potential variable clinical course of COVID-19 pneumonia, particularly in pregnancy.

The physiologic changes of pregnancy are important to account for in the setting of respiratory collapse and mechanical ventilation. The normal compensated respiratory alkalosis of pregnancy $\left(\mathrm{PCO}_{2} 28-32 \mathrm{~mm} \mathrm{Hg}\right)$ should influence the selected respiratory rate. Studies show that 'permissive hypercapnia' (up to $50 \mathrm{~mm}$ $\mathrm{Hg}$ ) has not been associated with adverse fetal effects. ${ }^{2}$ A target $\mathrm{PaO}_{2}$ of $70 \mathrm{~mm} \mathrm{Hg}$ is appropriate during pregnancy, in contrast to $55-80 \mathrm{~mm} \mathrm{Hg}$ in the non-pregnant state and facilitates maintenance of maternal oxygen saturation at greater than $95 \%$. These targets guide ventilator $\mathrm{FiO}_{2}$ parameters.

Fetal considerations, particularly in the periviable gestational age window, often complicate clinical decision making. The guiding principle that optimal management of maternal status is also optimal management for the fetus is too often not adhered to. We have too little experience with respiratory collapse requiring mechanical ventilation for COVID-19 pneumonia to determine if delivery (regardless of route) facilitates maternal resuscitation or hinders it.

Given these uncertainties, it is critical to have a conversation with the patient, or her surrogate decision maker (durable power of attorney) if the patient is incapacitated, regarding interventions for fetal indications, especially in patients at an early gestational age. Counseling should highlight the balance of risk and benefit for maternal status and fetal status but should accentuate the precept that a disassociation between maternal and fetal interests is rare. A multidisciplinary approach with neonatology consultation is also valuable under these critical circumstances to provide information regarding fetal prognosis and wishes for neonatal resuscitation in the periviable gestational age window. It is also important to discuss, and prepare for, the possibility of perimortem cesarean section if maternal cardiac arrest occurs.

Oral hydroxychloroquine has been recommended at our institution for all COVID-19 patients requiring treatment. The Maternal Fetal Medicine team counseled obstetric patients about hydroxychloroquine use in pregnancy when applicable. The American College of Obstetrics and Gynecology recommends use of hydroxychloroquine when indicated; it is a low-risk drug in pregnancy despite its ability to cross into placental circulation. ${ }^{3}$ It is uncertain whether our institution's empiric use of hydroxychloroquine and prednisone 
facilitated our patients' recovery. Currently, there is no treatment for COVID-19 approved by the U.S. Food and Drug Administration. Our institution's empiric use of hydroxychloroquine and prednisone is based upon novel studies that indicated its potential benefit as a treatment for severe COVID-19 infection. ${ }^{4-7}$

The use of proning appeared crucial in 2 of our patients. Placing an individual in the prone position allows for recruitment of alveoli and corrects ventilation perfusion mismatch, which occurs in acute respiratory distress syndrome. ${ }^{8}$ Patients selected for this treatment continued to be hypoxic despite optimizing mechanical ventilation in the supine position. The criteria for pregnant patients to be placed in prone positioning are the same as non-pregnant individuals. ${ }^{9}$ Clinicians previously were hesitant to place pregnant patients in the prone position because of uncertainty regarding fetal response. During the 2009 influenza pandemic, however, many pregnant patients underwent prone ventilation as rescue therapy. Case reports described successful proning in pregnancy without apparent adverse maternal or fetal effects. ${ }^{10}$

It is important to notice that many of the risk factors that increase the risk of severe COVID-19 infection in the non-pregnant patient are mirrored in the pregnant patient. Most critically ill patients in this case series were obese and African American. Review of the demographics of patients hospitalized with COVID-19 revealed that the African American population is disproportionately affected. ${ }^{11,12}$ Additionally, studies also illustrate an inverse correlation between age and BMI, indicating that younger patients with severe disease were more likely to be obese. ${ }^{13}$ These characteristics are imperative to identify those at high risk for severe disease given the potential for rapid clinical deterioration and need for mechanical ventilation.

\section{Conclusion}

In conclusion, this case series adds to the expanding literature describing the complex care surrounding pregnant patients with severe COVID-19 pneumonia requiring intensive care management. As the pandemic continues, we hope that our experience helps to inform the care of these critically ill patients.

\section{Disclosure statement}

The authors declare that they have no potential conflicts of interest.

\section{Author Contributions}

Nicolina Smith drafted the manuscript.

Leah Hong drafted the manuscript.

Madhurima Keerthy contributed to review and editing of the manuscript.

Monica Lee-Griffith contributed to review and editing of the manuscript.

Robyn Garcia contributed to review and editing of the manuscript.

Majid Shaman contributed to review and editing of the manuscript.

Gregory Goyert contributed to review and editing of the manuscript.

All authors contributed equally to creation of this case report.

\section{Ethics Statement}

No ethics board was required in this study. The study was approved by Henry Ford Health System's IRB.

\section{Funding Statement}

There was no financial support or sponsorship for this study.

\section{References}

1. Gattinoni L, Chiumello D, Caironi P, Busana M, Romitti F, Brazzi L, et al. COVID-19 pneumonia: different respiratory treatments for different phenotypes? Intensive Care Med 2020;Epub ahead of print. https://doi.org/10.1007/s00134-020-06033-2 
2. Ma J, Ye H. Effects of permissive hypercapnia on pulmonary and neurodevelopmental sequelae in extremely low birth weight infants: a meta-analysis. Springerplus 2016;5:764.

3. Sperber K, Hom C, Chao CP, Shapiro D, Ash J. Systematic review of hydroxychloroquine use in pregnant patients with autoimmune diseases. Pediatr Rheumatol Online J 2009;7:9.

4. Wang M, Cao R, Zhang L, Yang X, Liu J, Xu M, et al. Remdesivir and chloroquine effectively inhibit the recently emerged novel coronavirus (2019-nCoV) in vitro. Cell Res 2020;30:269-71.

5. Gao J, Tian Z, Yang X. Breakthrough: chloroquine phosphate has shown apparent efficacy in treatment of COVID-19 associated pneumonia in clinical studies. Biosci Trends 2020;14:72-3.

6. Colson P, Rolain JM, Lagier JC, Brouqui P, Raoult D. Chloroquine and hydroxychloroquine as available weapons to fight COVID-19. Int J Antimicrob Agents 2020;55:105932.

7. Russell B, Moss C, George G, Santaolalla A, Cope A, Papa S, et al. Associations between immunesuppressive and stimulating drugs and novel COVID-19 - a systematic review of current evidence. Ecancermedicalscience 2020;14:1022.

8. Corneji RA, Diaz JC, Tobar EA, Bruhn AR, Ramos CA, Gonzalez RA, et al. Effect of prone positioning on lung protection in patient with acute respiratory distress syndrome. Am J Respir Crit Care Med 2013;188:440-8.

9. Guerin C, Reignier J, Richard JC, Beuret P, Gacouin A, Boulain T, et al. Prone positioning in severe acute respiratory distress syndrome. N Engl J Med 2013;368:2159-68.

10. Samanta S, Samanta S, Wig J, Baronia AK. How safe is the prone position in acute respiratory distress syndrome at late pregnancy? Am J Emerg Med 2014;32:687.e1-3.

11. Garg S, Kim L, Whitaker M, O'Halloran A, Cummings C, Holstein R, et al. Hospitalization rates and characteristics of patients hospitalized with laboratory-confirmed coronavirus disease 2019 - COVID-NET, 14 states, March 1-30, 2020. MMWR Morb Mortal Wkly Rep 2020;69:458-64.

12. Gold JA, Wong KK, Szablewski CM, Patel PR, Rossow J, da Silva J, et al. Characteristics and clinical outcomes of adult patients hospitalized with COVID-19 - Georgia, March 2020. MMWR Morb Mortal Wkly Rep 2020;69:545-50.

13. Kass DA, Duggal P, Cingolani O. Obesity could shift severe COVID-19 disease to younger ages. Lancet 2020;395:1544-5.

Table 1. Patient demographics

\begin{tabular}{lll}
\hline Demographics & Case $\mathbf{1}$ & Case $\mathbf{2}$ \\
Age & 35 years old & 37 years old \\
Ethnicity & African American & Arab American \\
Gestational age at presentation & $30 \mathrm{w} 4 \mathrm{~d}$ & $23 \mathrm{w} 0 \mathrm{~d}$ \\
Comorbidities & Obesity & Obesity, well-controlled asthma, uncontrolled type 2 diabetes mellitus \\
BMI $\left(\mathrm{kg} / \mathrm{m}^{2}\right)$ & 35.9 & 56 \\
\hline
\end{tabular}

w, weeks; d, days; BMI, body mass index.

Table 2. Clinical Presentation

\begin{tabular}{ll}
\hline Clinical Presentation & Case 1 \\
Sick contact & Yes \\
Symptoms & Shortness of breath \\
Vitals & Tachycardia $(110 \mathrm{bpm})$, tachypnea (38 breaths $/ \mathrm{min})$, hypoxic $\left(\mathrm{SPO}_{2} 90 \%\right.$ room air $)$, and afebri
\end{tabular}


Chest radiography Interstitial opacities

Laboratory abnormalities

CRP, C-reactive protein; EKG, electrocardiogram.

Table 3. Treatment modalities

\begin{tabular}{lll}
\hline Treatment & Case 1 & Case 2 \\
COVID-19 treatment & IV methylprednisolone & Standard hydroxychloroquine and \\
Superimposed bacterial pneumonia & None & Ceftriaxone \\
VTE prophylaxis & Enoxaparin 40 mg SQ daily & Heparin 5,000 U SQ every 8 hour \\
Obstetrics interventions & Betamethasone 12 mg IM, 2 doses 24 hours apart & Betamethasone 12 mg IM, 2 dose \\
Miscellaneous & None & None \\
\hline
\end{tabular}

IV, intravenous; VTE, venothromboembolus; SQ, subcutaneous; IM, intramuscular.

Table 4. Hospital course

\begin{tabular}{lll}
\hline Hospital Course & Case 1 & Case 2 \\
Respiratory support & High flow nasal cannula & Mechanical ventilation \\
Days intubated & 0 & 14 \\
Maximum ventilatory settings & $\mathrm{N} / \mathrm{A}$ & PEEP $18 \mathrm{~cm} \mathrm{H}_{2} \mathrm{O}, \mathrm{FiO}_{2} 100 \%$ \\
Days hospitalized & 9 & 17 \\
Delivery & No & No \\
Outcome & Discharged on room air. & Patient left against medical advice on hospital day 17 and was c \\
\hline
\end{tabular}

N/A, not available ; PEEP, positive end-expiratory pressure ; FHT, fetal heart tracing; TID, three times a day. 